\title{
A role of protein degradation in memory consolidation after initial learning and extinction learning in the honeybee (Apis mellifera)
}

\author{
Johannes Felsenberg, Vincent Dombrowski, and Dorothea Eisenhardt ${ }^{1}$ \\ FB Biologie, Pharmazie, Chemie, Institut für Biologie, Neurobiologie, Freie Universität Berlin, 14195 Berlin, Germany
}

\begin{abstract}
Protein degradation is known to affect memory formation after extinction learning. We demonstrate here that an inhibitor of protein degradation, MGl32, interferes with memory formation after extinction learning in a classical appetitive conditioning paradigm. In addition, we find an enhancement of memory formation when the same inhibitor is applied after initial learning. This result supports the idea that MG132 targets an ongoing consolidation process. Furthermore, we demonstrate that the sensitivity of memory formation after initial learning and extinction learning to MG132 depends in the same way on the number of CS-US trials and the intertrial interval applied during initial learning. This supports the idea that the learning parameters during acquisition are critical for memory formation after extinction and that protein degradation in both learning processes might be functionally linked.
\end{abstract}

In classical conditioning, animals learn to associate a conditioned stimulus (CS) with an unconditioned stimulus (US). Memories of varying stability are formed about this association that differ in molecular mechanisms underlying their induction and maintenance; short-lasting memories depend on the modification of neuronal proteins, i.e., phosphorylation, whereas long-lasting memories undergo a transcription- and translation-dependent process of stabilization. This stabilization process is termed memory consolidation (Dudai 2004). Besides the de novo synthesis of proteins, protein degradation might be critical for consolidation. Several investigators have demonstrated an involvement of protein degradation in memory formation and proposed that protein degradation plays a critical role in ongoing memory consolidation after learning (Lopez-Salon et al. 2001; Yeh et al. 2006; Merlo and Romano 2007; Artinian et al. 2008; Jarome et al. 2011; Kottler et al. 2011; Rodriguez-Ortiz et al. 2011).

Protein degradation is also critical for memory formation following the retrieval of a consolidated memory without reinforcement, i.e., during reconsolidation and the consolidation of an extinction memory (Artinian et al. 2008; Lee et al. 2008; Jarome et al. 2011). During reconsolidation, a previously consolidated memory is stabilized after it has been reactivated and destroyed to enable memory updating and reorganisation after retrieval (Nader and Hardt 2009). During extinction, animals learn that the previously reinforced CS is not reinforced anymore and form a memory about this CS-noUS association (Myers and Davis 2002). Synaptic proteins are degraded after memory retrieval, and inhibitors of protein degradation affect reconsolidation and the formation of a long-term extinction memory (Lee et al. 2008; Jarome et al. 2011). Therefore, it has been proposed that following retrieval protein degradation destroys a previously consolidated memory to enable its updating and reorganization (Lee et al. 2008). Taken together, the data indicate that protein degradation might play a different role following initial learning and memory retrieval: Following initial learning, it might be involved in an ongoing consolidation process, whereas following memory retrieval, it might contribute to the destruction of the retrieved memory.

\footnotetext{
'Corresponding author

E-mail dorothea.eisenhardt@fu-berlin.de

Article is online at http://www.learnmem.org/cgi/doi/10.1101/lm.026245.112.
}

However, it is unclear if this proposal holds true, because it is in contrast to results from behavioral studies on extinction. Three behavioral phenomena described in conjunction with extinction-i.e., spontaneous recovery, renewal, and reinstatementdemonstrate a time- and context-dependent reappearance of the conditioned response after extinction took place (Myers and Davis 2002). This suggests that extinction does not cause a destruction of the extinguished memory but the formation of a parallel memory about the CS-noUS association, leaving the extinguished CS-US memory intact (Bouton 2004). Thus, following extinction, labilization of the extinguished memory might not be necessary. In line protein degradation would not be involved in such a process. In addition, Jarome et al. (2011) reported that the same proteins are degraded after initial learning and memory retrieval. This might suggest a similar role for protein degradation following initial learning and memory retrieval.

Thus the role of protein degradation in memory consolidation is still unclear. To gain insight into the role of protein degradation in memory consolidation, here we tested two hypotheses: (1) Protein degradation plays a role in an ongoing consolidation process; (2) protein degradation plays a role after retrieval of consolidated memories.

In an appetitive conditioning paradigm in the honeybee (Apis mellifera), we studied the sensitivity of memory formation to an inhibitor of protein degradation after initial learning and after extinction learning. After pairing an odor (CS) with a sucrose reward (US), honeybees learn the association between the CS and the US and form a memory about this association (Bitterman et al. 1983; Giurfa and Sandoz 2012). One day after this initial learning process, the presentation of two CS trials alone leads to extinction learning and the formation of a consolidated extinction memory (Stollhoff et al. 2005; Stollhoff and Eisenhardt 2009).

We demonstrated that an inhibitor of protein degradation, MG132, affects memory formation following initial learning and extinction learning. We observed sensitivity to MG132, when learning parameters were used that are known to induce memory consolidation. We show that the sensitivity of memory formation after initial learning and extinction learning depends in the same way on the number of CS-US trials and the intertrial interval applied during acquisition. This demonstrates that the 
learning parameters during acquisition are critical for memory formation after extinction and that protein degradation in both learning processes might be functionally linked.

\section{Results}

\section{Memory consolidation after extinction learning is sensitive to the protease inhibitor MG132}

We first asked whether protein degradation is needed for the consolidation of an extinction memory. Stollhoff et al. (2005) demonstrated that extinguishing a consolidated olfactory memory with two extinction trials leads to a consolidated extinction memory 1 d later. Therefore, we first tested the effect of an inhibitor of protein degradation on retention of this extinction memory. We used Z-Leu-Leu-Leu-Al (MG132), an inhibitor of proteases, namely, the proteasome, calpain, and cathepsin (Kisselev and Goldberg 2001) that has been demonstrated to inhibit protein degradation in insects (Naidoo et al. 1999; Imai et al. 2005; Lundgren et al. 2005; Katsuma et al. 2011).

Honeybees were conditioned with three CS-US trials with an intertrial interval (ITI) of $10 \mathrm{~min}$ on day 1 . They were divided $1 \mathrm{~d}$ later into two groups: animals that received two CS trials alone (= extinction trials) with an ITI of $10 \mathrm{~min}$ and animals that did not receive the extinction trials. One hour after extinction onset, animals were subdivided into animals that were injected with MG132 and animals that were injected with PBS. One day later, on day 3, memory retention of all animals was tested with a CS presentation alone (Fig. 1A).

Irrespective of MG132 or PBS injection, we observed a significant difference between the first extinction trial on day 2 and the retention test on day 3 (McNemar $_{\mathrm{PBS}}: x=23.36, P<0.05$; McNemar $_{\text {MG132: } \chi}=4.36, P<0.05$ ) (Fig. 1B). Thus, in these animals, long-term extinction was observed on day 3 . However, a significant difference between the CRs of the MG132- and the PBS-treated animals was only observed (G-Test: PBS $_{\text {retrieved }}$ vs. MG132 $2_{\text {retrieved, }} G=5.74, P<0.05$ ) in animals that received the extinction trials but not in animals that were not subjected to extinction trials (G-Test: $\mathrm{PBS}_{\text {unretrieved }}$ vs. MG132 $2_{\text {unretrieved, }} G=0.49$, $P>0.05$ ) (Fig. 1B,C).

Taken together, we conclude that consolidation of an extinction memory is sensitive to MG 132. This supports the idea that MG132 inhibits a degradation process that is induced by extinction and labilizes the previously consolidated memory.

\section{Memory formation after extinction is not sensitive to MGl32 when the extinguished memory did not undergo a consolidation process}

If protein degradation induced by extinction serves to destabilize a consolidated memory, the inhibitor should solely affect extinction of this consolidated memory. The inhibitor should not target extinction of a memory that did not undergo a consolidation process.

Friedrich et al. (2004) demonstrated that protein synthesisdependent memory consolidation does not take place when honeybees are conditioned with only one trial. Next, we tested whether formation of extinction memory after one trial of initial learning is affected by MG132.

We treated animals as in the first experiment but trained them with one instead of three conditioning trials on day 1 .

Honeybees were conditioned with one CS-US trial on day 1. They were divided into two groups. One day later (day 2), one group received two extinction trials; the other group did not. One hour after extinction, both groups were subdivided into one group that was injected with MG132 and one group that
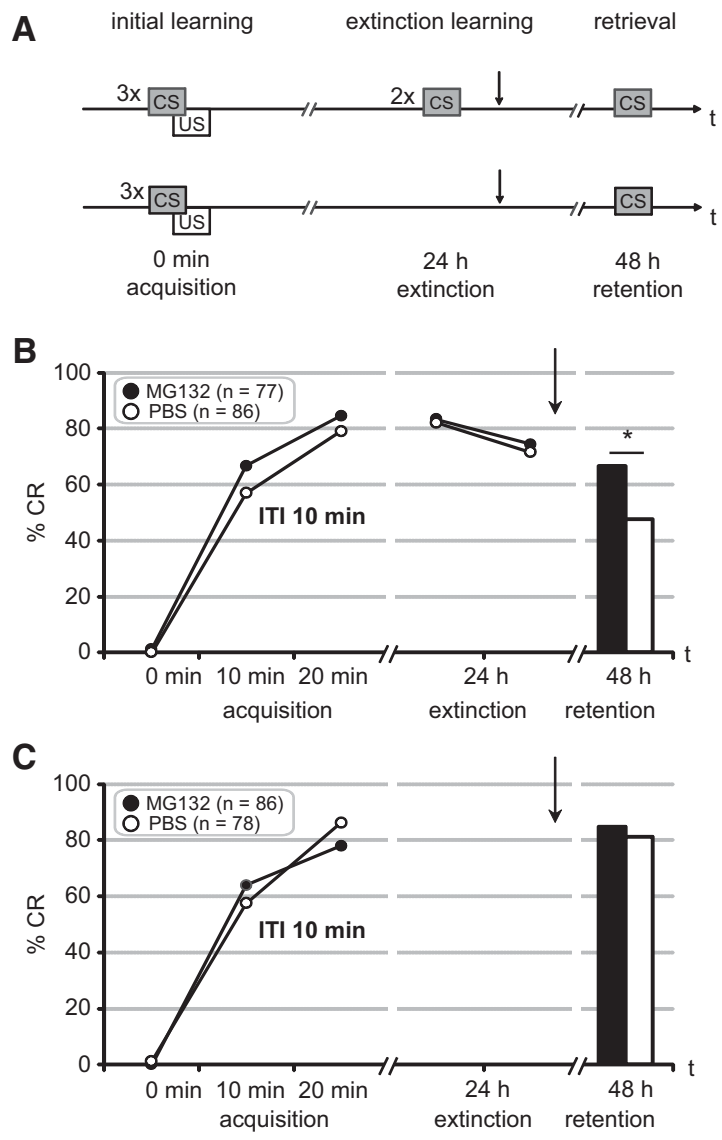

Figure 1. Memory formation after extinction learning is sensitive to MG132. (A) Diagram of the experiment shown in B and C: The experiment was conducted on three consecutive days. On the first day, animals were subjected to three CS-US trials with $10 \mathrm{~min} \mathrm{ITI.} \mathrm{On} \mathrm{the} \mathrm{fol-}$ lowing day, bees were divided into one group that received two extinction trials with an ITI of $10 \mathrm{~min}(B)$ and another group that did not receive extinction trials $(C)$. One hour after starting extinction, animals of both groups were injected with MG132 (black) or PBS (white). On the third day, memory retention was tested. (Arrow) The time point of injection. Acquisition, extinction, and retention tests depict the three phases of the experiment during which initial learning, extinction learning, and retrieval take place. $(B)$ In the extinguished group, the difference of $\mathrm{CR}$ at the retention test $1 \mathrm{~d}$ after extinction between the MG132-treated and the PBS-treated animals was significant. (C) The difference of $C R$ at the retention test between the MG132-treated and the PBS-treated animals was not significant, when extinction was omitted. $\left.{ }^{*}\right)$ Significant differences $(P<0.05)$ between groups.

was injected with PBS. One day later (day 3), we tested memory retention of all animals (Fig. 2A).

In both groups of bees (MG132 and PBS) that received two extinction trials on day 2, the difference between the first extinction trial on day 2 and the retention test on day 3 was significant $\left(\right.$ McNemar $_{\mathrm{PBS}}: \chi=8.64, P<0.05$; McNemar $_{\mathrm{MG} 132}: \chi=8.45, P<$ 0.05) (Fig. 2B). Accordingly, long-term extinction was observed on day 3 in the PBS group and the MG132 group. However, a significant difference between the retention scores of the MG132- and PBS-treated animals on day 3 was not observed (PBS $_{\text {retrieved vs. MG132 }}$ retrieved: $G=0.1, P>0.05$ ) (Fig. 2B). A significant difference between retention scores was also not observed between animals of the PBS group and the MG132 group that were not subjected to the two extinction trials $\left(\mathrm{PBS}_{\text {unretrieved }}\right.$ vs. MG132 unretrieved: $G=0.35, P>0.05$ ) (Fig. 2C). Thus, MG132 did not inhibit extinction memory retention on day 3. 


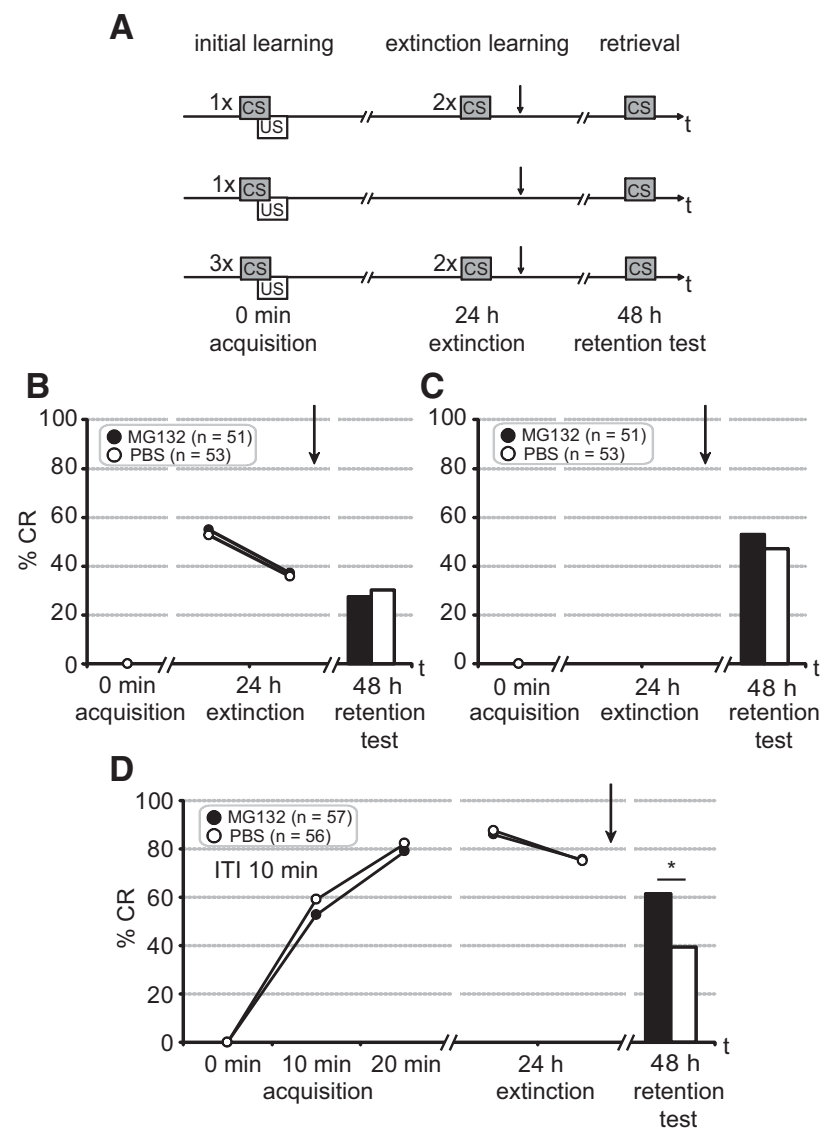

Figure 2. Memory formation after extinction learning is not sensitive to MG132 after presentation of one CS-US trial during acquisition. (A) Diagram of the experiments shown in $B-D$ : On the first day, animals were subjected to one CS-US trial or three CS-US trials with 10 min ITI. On the following day, animals that were conditioned with one CS-US trial were divided into one group that received two extinction trials with an ITI of $10 \mathrm{~min}(B)$ and another group that did not receive these trials (C). All animals that were conditioned with three CS-US trials received two extinction trials $(D)$. One hour after starting extinction, the animals were injected with MG132 (black) or PBS (white). On the third day, memory was tested. (Arrow) The time point of injection. Acquisition, extinction, and retention test depict the three phases of the experiment during which initial learning, extinction learning, and retrieval take place. $(B, C)$ After presentation of one CS-US trial during acquisition, no differences of the $C R$ at the retention test between the MG132-treated and the PBS-treated group was observed irrespective of whether the animals were extinguished or not. $(D)$ In animals that were conditioned with three CS-US trials, a significant difference was seen of the CR between the MG132 and the PBS group at the retention test $1 \mathrm{~d}$ after extinction. $\left({ }^{*}\right)$ Significant differences $(P<0.05)$ between groups.

The negative result of this experiment might be explained by the fact that the inhibitor was not active in this experiment. Therefore, we ran an experiment in parallel where we conditioned the animals with three trials with an ITI of $10 \mathrm{~min}$ (Fig. 2A). In this experiment, we used the same stock of MG132 as in the previous two experiments and demonstrated a significant difference between retention scores after extinction between the MG132 and

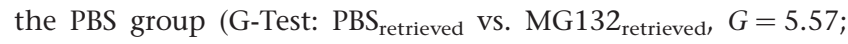
$P<0.05$ ) (Fig. 2D).

Taken together, our data demonstrate that MG132 does not affect memory formation after extinction learning when the animals have been exposed to one conditioning trial during initial learning. Hence, MG132 application shortly after extinction learning might affect only a consolidated CS-US memory. This interpretation is consistent with the idea that protein degradation after extinction serves to destabilize a consolidated memory but not a memory that did not undergo consolidation. However, an alternative explanation could be an effect of MG132 on an ongoing consolidation, i.e., reconsolidation or the consolidation of the extinction memory.

\section{The protease inhibitor MG132 enhances memory formation after initial learning}

Next, we asked whether MG132 affects only consolidated memory or rather an ongoing consolidation process. Therefore, we examined the effect of MG132 applied shortly after initial learning. After initial learning with three trials, memory consolidation is sensitive to protein synthesis inhibitors applied shortly after acquisition (Friedrich et al. 2004; Felsenberg et al. 2011). Hence, the consolidation process should not yet be finalized. If MG132 affects only already-consolidated memory, this ongoing consolidation process should not be sensitive to MG132.

We conditioned honeybees with three CS-US trials with an ITI of $10 \mathrm{~min}$. One hour after the onset of conditioning, the animals were divided into two groups that were injected with either MG132 or PBS. Memory retention was tested cumulatively after $1 \mathrm{~d}, 2 \mathrm{~d}$, and $3 \mathrm{~d}$ (Fig. 3A). Retention scores differed significantly over all three days between the MG132- and the PBS-treated animals (rmANOVA factor group: $F_{(2,106)}=11.58$; $P<0.05$ ) (Fig. 3B).

Thus we demonstrate that MG132 enhances memory formation when injected shortly after initial learning with three conditioning trials and an ITI of $10 \mathrm{~min}$. This does not support our idea that MG132 affects solely already-consolidated memories. Rather, it suggests that MG132 affects memories while they are consolidating.

\section{MG132 affects an ongoing consolidation process}

Above we observed an effect of MG132 on a long-term memory although it has been applied in a situation where consolidation is not yet finalized. This might indicate that MG132 targets a
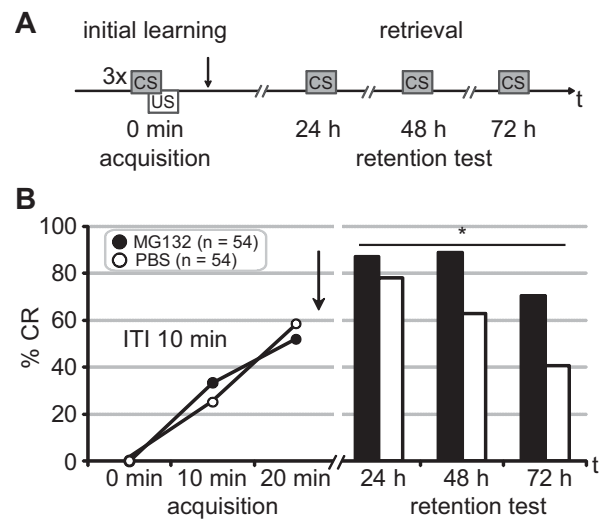

Figure 3. MG132 sensitivity of memory formation after initial learning. $(A)$ Diagram of the experiment shown in $B$ : Honeybees were conditioned with three CS-US trials with an ITI of 10 min, injected with MG132 (black) or PBS (white) $1 \mathrm{~h}$ after conditioning starts, and tested on three consecutive days after conditioning. (Arrow) The time point of injection. Acquisition and retention test depict the two phases of the experiment during which initial learning and retrieval take place. $(B)$ The percentage of CR was significantly different between the MG132- and the PBS-treated bees 1, 2, and $3 \mathrm{~d}$ after acquisition. (*) A significant difference for the rmANOVA $(P<0.05)$ between groups. 
memory that is still in the process of consolidation. To test this idea, we next asked whether the observed memory enhancement by MG132 after initial learning could only be observed for a consolidating memory. Therefore, we ran two experiments in parallel. We conditioned honeybees with a second protocol that induces a consolidated memory (Wüstenberg et al. 1998; Friedrich et al. 2004) and with a protocol that does not induce memory consolidation (Friedrich et al. 2004), and applied MG132 again shortly after the initial learning process.

In the first experiment, animals were conditioned with three CS-US trials with an ITI of $2 \mathrm{~min}$. One hour later, these animals were divided into two subgroups that were injected with either MG132 or the solvent PBS. Memory retention was tested cumulatively $1 \mathrm{~d}, 2 \mathrm{~d}$, and $3 \mathrm{~d}$ after conditioning (Fig. 4A1).

Memory retention $1 \mathrm{~d}, 2 \mathrm{~d}$, and $3 \mathrm{~d}$ after conditioning was increased in the MG132 group compared with the PBS group (three trials; rmANOVA: $\left.F_{(1,98)}=4.88 ; P<0.05\right)$ (Fig. 4 A2).

In the second experiment, animals were conditioned with one CS-US trial. One hour later, these animals were divided into two subgroups that were injected with either MG132 or PBS. Memory retention was tested cumulatively $1 \mathrm{~d}, 2 \mathrm{~d}$, and $3 \mathrm{~d}$ after conditioning (Fig. 4B1).

Memory retention was not significantly different between the MG132 and the PBS groups (single trial; rmANOVA: $F_{(1,89)}=$ 0.68; $P>0.05$ ) (Fig. 4B2).

Taken together, our data demonstrate that MG132 affects memory formation shortly after initial learning only after a conditioning protocol known to induce a consolidation process. This supports the idea that MG132 affects an ongoing consolidation process. Given that MG132 targets the same processes after initial learning and extinction learning, as it has been described for inhibitors of protein synthesis (Lattal et al. 2006), we conclude that MG132 might target an ongoing consolidation process not only after initial learning but also after extinction learning.

\section{Memory formation after extinction is sensitive to MG132 only, if this applies to memory formation after initial learning}

Above we demonstrate that memory formation after initial learning and after extinction learning are sensitive to MG132 when three conditioning trials are presented during acquisition learning. When one conditioning trial is presented during initial learning, a sensitivity of memory formation after initial learning and extinction learning could not be shown. We conclude that the sensitivity of memory formation after initial learning and extinction learning to MG132 might depend in the same way on the conditioning protocol during initial learning. This might indicate that protein degradation during consolidation of an acquisition memory and protein degradation during consolidation of an extinction memory are functionally linked.

To test this idea, we varied the conditioning protocol during initial learning and kept the parameters during extinction learning constant. We presented two conditioning trials with an ITI of
$10 \mathrm{~min}$ or $2 \mathrm{~min}$ during acquisition. If protein degradation after initial learning and extinction learning are functionally linked, the same conditioning protocol applied during acquisition should lead to a MG132-sensitive memory formation after acquisition learning and to a MG132-sensitive memory formation after extinction learning.

In the first experiment, we tested the effect of MG132 on memory formation after initial learning applying two different conditioning protocols during acquisition. Two groups of honeybees were conditioned with two CS-US trials. The first group was conditioned with an ITI of $10 \mathrm{~min}$, the second group with an ITI of $2 \mathrm{~min}$. One hour after the first conditioning trial, both groups were injected with either MG132 or PBS. Memory retention of both groups was tested after $1 \mathrm{~d}, 2 \mathrm{~d}$, and $3 \mathrm{~d}$ (Fig. 5A). In the first group, conditioned with an ITI of $10 \mathrm{~min}$, the difference between the MG132- and the PBS-treated animals is significant $1 \mathrm{~d}$ after conditioning (rmANOVA: group $\times$ time, $F_{(2,172)}=12.36 ; P<$ 0.05 ; Fisher's LSD post hoc test day $_{1}$ MG132 vs. PBs: $\left.P<0.05\right)$. In the second group, conditioned with an ITI of $2 \mathrm{~min}$, no significant difference is visible (rmANOVA: group $\times$ time, $F_{(2,144)}=0.55 ; P>$ 0.05) (Fig. 5B,C).

Taken together our data demonstrate that training with an ITI of 10 min but not an ITI of 2 min results in an MG132-dependent enhancement of memory formation after initial learning.

Next, we applied the same two conditioning protocols during acquisition as in the previous experiment but tested the effect of MG132 on memory formation after extinction. Extinction was performed $1 \mathrm{~d}$ after conditioning with two trials with an ITI of $10 \mathrm{~min}$ on day 1. Afterward honeybees were divided into two groups. One group received two extinction trials; the other group did not. One hour after extinction onset, both groups were subdivided into animals that were injected with MG132 or with PBS. One day later, on day 3, retention of all animals was tested (Fig. $6 \mathrm{~A})$. On day 2 , the difference between the first extinction trial on day 2 and the retention test on day 3 was significant in PBStreated bees that received extinction trials $\left(\operatorname{McNemar}_{\mathrm{PBS}}: \chi=\right.$ 9.38; $P<0.05)$ but not in MG132-treated bees $\left(\right.$ McNemar $_{\text {MG132: }}$ :
A1

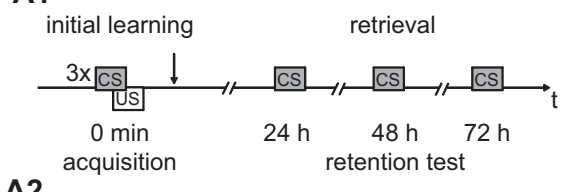

A2

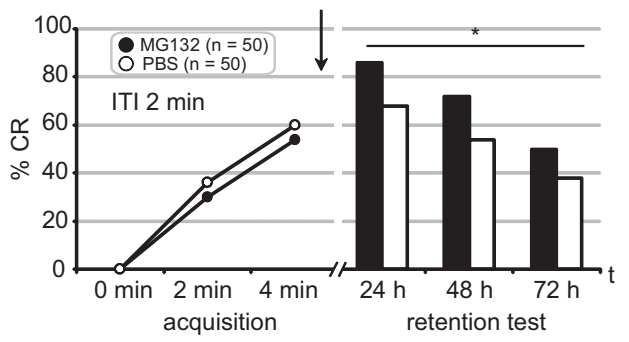

B1

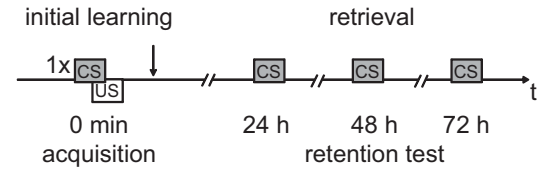

B2

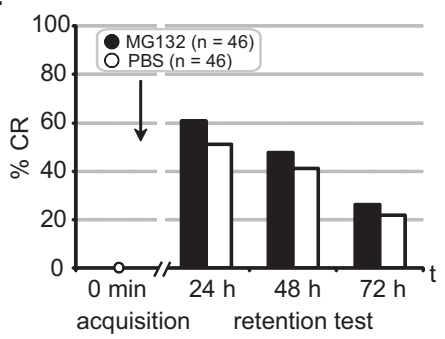

Figure 4. MG132 sensitivity of memory formation after initial learning depends on the number of CS-US trials presented during acquisition. $(A 1, B 1)$ Diagram of the experiments shown in $A 2$ and $B 2$ : Honeybees were conditioned with three CS-US trials with an ITI of 2 min (A1) or with one CS-US trial (B1). Both groups were injected with MG132 (black) or PBS (white) $1 \mathrm{~h}$ later, and tested on three consecutive days. (Arrow) The time point of injection. Acquisition and retention tests depict the two phases of the experiment during which initial learning and retrieval take place. (A2) The percentage of CR was significantly different between the MG132- and the PBS-treated bees when tested 1, 2, and $3 \mathrm{~d}$ after presentation of three CS-US trials during acquisition. (B2) No significant difference of CR was observed between the MG132- and PBS-injected bees when tested 1, 2, and $3 \mathrm{~d}$ after presentation of one CS-US trial during acquisition. $\left(^{*}\right)$ A significant difference for the rmANOVA $(P<0.05)$ between groups. 
A

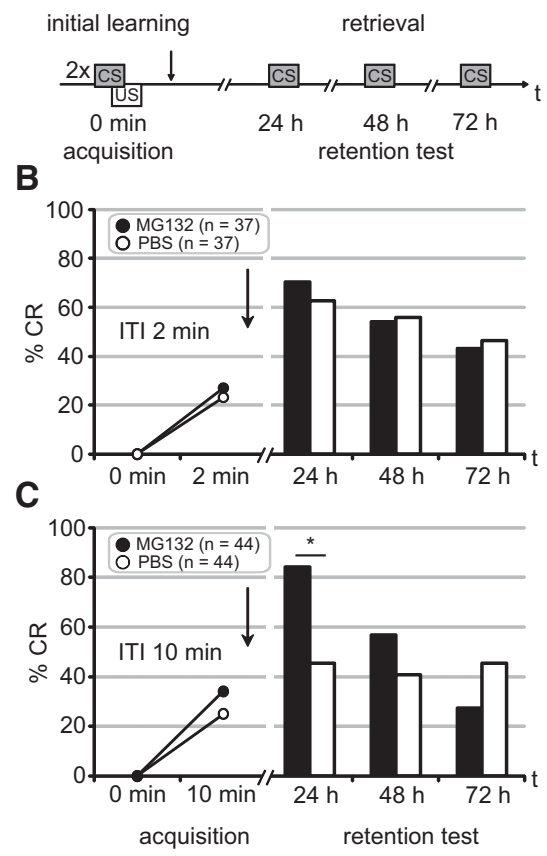

Figure 5. Sensitivity of memory formation after initial learning to MG132 depends on the ITI between CS-US trials. (A) Diagram of the experiments shown in $B$ and $C$ : The experiments were conducted on four consecutive days. On the first day, animals were subjected to two CS-US trials with either 2 min ITI (B) or 10 min ITI (C). One hour after conditioning started, animals were injected with either MG132 (black) or PBS (white). Memory tests were performed on three consecutive days. Acquisition and retention tests depict the two phases of the experiment during which initial learning and retrieval take place. (Arrow) The time point of injection. (B) No significant difference of CR was observed between the MG132- and PBS-injected bees when tested 1, 2, and $3 \mathrm{~d}$ after presentation of two CS-US pairings with an ITI of 2 min during acquisition. (C) The percentage of CR was significantly different between the MG132- and the PBS-treated bees when tested 1, 2, and $3 \mathrm{~d}$ after presentation of two CS-US pairings with an ITI of 10 min during acquisition. $\left({ }^{*}\right) A$ significant difference for the rmANOVA $(P<0.05)$ for group $\times$ time point and post hoc test revealed a significant difference for day 1 .

$\chi=0.24 ; P>0.05$ ) (Fig. 6B1,2). Accordingly, long-term extinction was observed on day 3 in the PBS group but not in the MG132 group.

On day 3, a significant difference between the CRs of the MG132 group and the PBS group was observed (G-Test: $\mathrm{PBS}_{\text {retrieved }}$ vs. MG132 $2_{\text {retrieved, }} G=5.89 ; P<0.05$ ) (Fig. 6B1). This difference was due to an increase of the CR in the MG132-treated group compared with the PBS-treated group. A significant difference between the MG132-treated and the PBS-treated bees was not demonstrated in the groups not subjected to the extinction trials (G-Test: PBS $_{\text {unretrieved vs. MG132 }}$ unretrieved, $G=0.44 ; P>0.05$ ) (Fig. 6 B2).

Thus, after initial learning with two trials and a 10 min ITI, memory formation after extinction learning is sensitive to MG132. This result resembles the effect of MG132 on memory formation after initial learning when applying the same conditioning protocol.

Next, we asked if an effect of MG132 on memory formation after extinction learning can be observed after initial learning with two trials and a 2 min ITI. After conditioning on day 1, honeybees were divided into two groups. On day 2 , one group received two extinction trials; the other group did not receive these trials.
One hour after extinction, both groups were subdivided into one group that was systemically injected with MG132 and one group that was injected with PBS. On day 3, memory retention of all animals was tested (Fig. 6A).

In animals exposed to two extinction trials on day 2 , a significant difference between the first extinction trial on day 2 and the retention test on day 3 was observed in the MG132 and PBS groups $\left(\right.$ McNemar $_{\mathrm{PBS}}: \chi=8.64, P<0.05$; McNemar $_{\mathrm{MG} 132}: \chi=8.45, P<$ 0.05) (Fig. 6C1,2). Thus, long-term extinction was observed on day 3 in both the PBS and the MG132 group. However, at the retention test on day 3, a significant difference between the CRs of the MG132-treated group and the PBS-treated group was not observed ( $\mathrm{PBS}_{\text {retrieved }}$ vs. MG132 $2_{\text {retrieved, }} G=0.5, P>0.05$ ) (Fig. 6C1). This also holds true for animals that did not receive extinction trials at day $2\left(\mathrm{PBS}_{\text {unretrieved }}\right.$ Vs. MG132 $2_{\text {unretrieved, }} G=0.05$, $P>0.05$ ) (Fig. 6C2). Thus, in this experiment, memory formation after extinction learning was not sensitive to MG132.

In a parallel experiment in which we used the same stock solution of MG132, we excluded that the inhibitor was not active in this experiment. We demonstrated a significant difference in memory retention after extinction between the MG132 and the PBS group after conditioning with three CS-US trials with an ITI of $10 \mathrm{~min}$ (G-Test: $\mathrm{PBS}_{\text {retrieved }}$ vs. MG132 retrieved, $_{\text {, }} G=4.62, P<$ 0.05) (Fig. 6D).

In summary, memory formation after initial learning and extinction learning is sensitive to MG132 when two trials with an ITI of 10 min were presented during acquisition. However, when the ITI during acquisition was $2 \mathrm{~min}$ instead of $10 \mathrm{~min}$, memory formation after initial learning or after extinction learning was not affected by MG132 injection. We therefore conclude that memory formation after extinction learning is only sensitive to MG132 if the process of memory formation of the extinguished memory is also sensitive to the inhibitor. This supports our idea that protein degradation after initial learning and protein degradation after extinction learning are functionally linked.

Taken together our results support the idea that after extinction MG132 targets an ongoing consolidation processes. MG132 affects memory consolidation after initial learning and after extinction learning. The sensitivity of the respective consolidation processes to MG132 depends in the same way on the number of trials and the ITI applied during initial learning. This result supports the idea that an up-to-date unknown process functionally links protein degradation during memory consolidation after initial learning and extinction learning.

\section{Discussion}

An ongoing consolidation process is sensitive for MG132 after initial learning and extinction learning

In this study, we have analyzed the role of protein degradation in extinction memory formation in the honeybee (A. mellifera). We used MG132, a reversible inhibitor of proteases (Kisselev and Goldberg 2001). We demonstrate that memory formation after extinction learning is sensitive to MG132 applied $1 \mathrm{~h}$ after extinction onset. We observed sensitivity to MG132 following extinction, when learning parameters were used that are known to induce memory consolidation in honeybees. In the first place, this supports the current idea by Lee et al. (2008) that protein degradation destroys a consolidated memory following extinction to enable updating and reorganization. However, we also demonstrate that MG132 applied $1 \mathrm{~h}$ after the onset of initial learning enhances long-term memory formation. Several studies in the honeybee demonstrate that $1 \mathrm{~h}$ after the onset of acquisition, memory consolidation is not yet finalized (Wüstenberg et al. 1998; Felsenberg et al. 2011). This supports the idea that after 

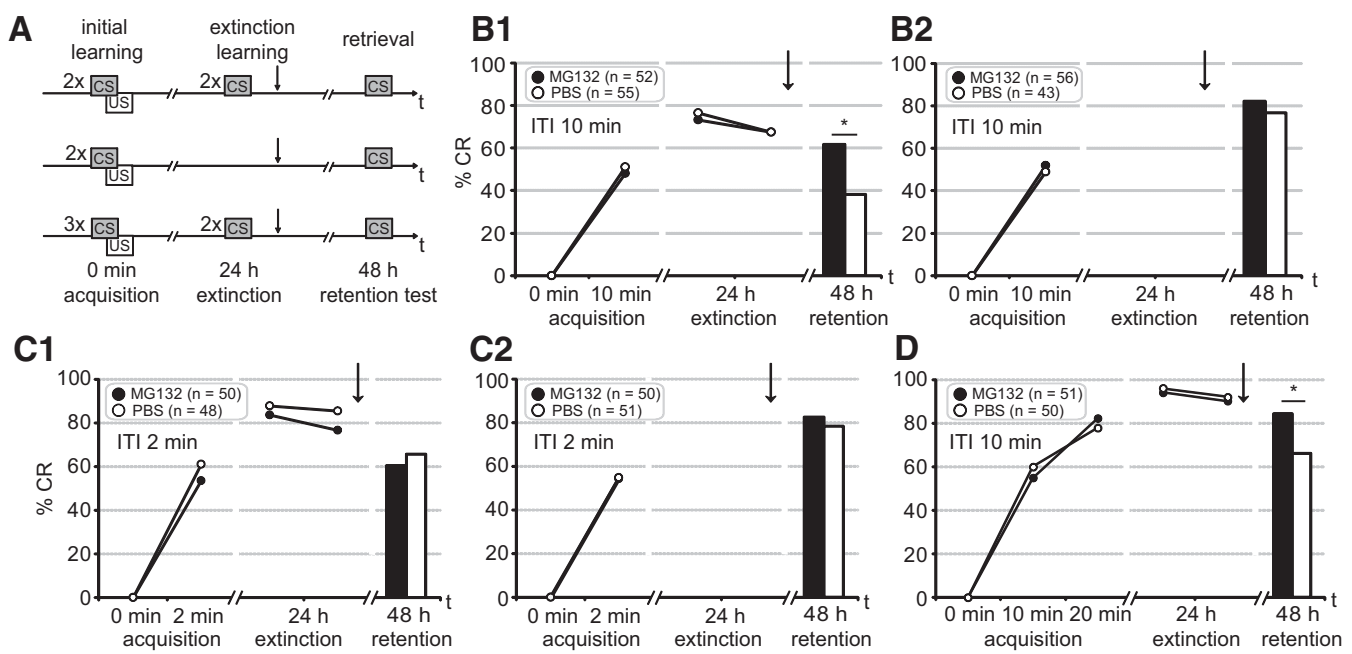

Figure 6. Sensitivity of memory formation after extinction learning to MG132 depends on the ITI between CS-US trials. $(A)$ Diagram of the experiments shown in $B 1, B 2, C 1, C 2$, and $D$ : On the first day, animals were subjected to two CS-US trials with 10 min ITI $(B 1, B 2)$ or 2 min ITI (C1, C2). On the following day, animals were divided into a group that received two extinction trials with an ITI of 10 min $(B 1, C 1)$ and one group that did not receive these trials $(B 2, C 2)$. In parallel animals in the control group were conditioned with three CS-US trials on the first day and received two extinction trials on the following day $(D)$. One hour after starting extinction, all groups were injected with MG132 (black) or PBS (white). On the third day, memory was tested. Acquisition, extinction, and retention tests depict the three phases of the experiment during which initial learning, extinction learning, and retrieval take place. (Arrow) The time point of injection. (B1) After presentation of two CS-US trials with an ITI of 10 min during acquisition, the difference of CR between the MG132- and the PBS-treated animals at the retention test $1 \mathrm{~d}$ after extinction was significant. (B2) When extinction was omitted, the difference between of CR between the MG132- and the PBS-treated animals at the retention test was not significant. (C1) When two CS-US trials were presented during acquisition with an ITI of $2 \mathrm{~min}$, the difference of CR between the MG132- and the PBS-treated animals at the retention test $1 \mathrm{~d}$ after extinction was not significant. (C2) When extinction was omitted, the difference between the percentage of CR in the MG132- and the PBS-treated group at the retention test was not significant. (D) After presentation of three CS-US trials with an ITI of 10 min during acquisition, the difference between the percentage of CR in the MG132- and the PBS-treated group at the retention test $1 \mathrm{~d}$ after extinction was significant. $\left(^{*}\right) \mathrm{A}$ significant difference for the rmANOVA $(P<0.05)$ between groups.

initial learning, an ongoing consolidation process is affected by MG132. Based on our findings, we cannot exclude that MG132 also interferes with an ongoing consolidation process following extinction learning. Accordingly, extinction learning might not rely on the destruction of the extinguished memory trace. Rather, protein degradation might underlie an ongoing consolidation process after extinction learning.

It remains unclear which consolidation process could be affected after extinction learning. Two processes have to be considered: reconsolidation or the consolidation of an extinction memory. We have demonstrated that MG132 enhances memory formation after initial learning. If MG132 also enhances extinction memory formation, a decrease of the retention score after extinction learning below the level of the control group should be observed. We do not see this effect. In contrast, we observe the opposite-namely, an enhancement of the retention score in the MG132 group compared with the control group (Fig. 1). This result supports the idea that in our study reconsolidation rather than the formation of an extinction memory is targeted by MG132 after extinction. MG132 might stabilize the reconsolidated memory, thereby shifting the balance from extinction memory toward the extinguished memory.

\section{MG132 enhances memory formation after initial learning}

The MG132-dependent enhancement of memory formation after initial learning concurs with findings in rats where MG132 injected into the amygdala enhances memory formation in the fearpotentiated startle paradigm (Yeh et al. 2006), suggesting that MG132-sensitive protein degradation constrains the formation of a memory trace after acquisition. Interestingly, in the sea hare, Aplysia california, an MG132-dependent enhancement of the synaptic strength has been described (Zhao et al. 2003), indicating that protein degradation constrains synaptic plasticity, a mechanism that might underlie the observed constraint of memory formation after acquisition.

However, Merlo and Romano (2007) demonstrated the opposite effect in the crab Chasmagnathus, namely, an inhibition of memory formation after application of MG132. In addition, studies that use other inhibitors of the proteasome documented an inhibition of memory formation and synaptic plasticity (Chain et al. 1999; Lopez-Salon et al. 2001; Fonseca et al. 2006; Karpova et al. 2006; Merlo and Romano 2007; Artinian et al. 2008). These studies conclude that protein degradation by the proteasome is necessary to degrade proteins that constrain long-lasting synaptic plasticity and the formation of long-term memory. However, a study on long-term plasticity demonstrated that both the degradation of synaptic constraints and the degradation of proteins facilitating synaptic strength might take place, suggesting that the proteasome inhibits the induction but facilitates the maintenance of synaptic plasticity (Dong et al. 2008). Accordingly, it might well be that in our study MG132 targets memory formation rather than memory maintenance.

However, it should be taken into account that in vertebrates, MG132 inhibits not only the proteasome but also calpain and cathepsin, albeit with a 10 times lower affinity (Kisselev and Goldberg 2001). Therefore, we cannot exclude that inhibition of calpain and cathepsin may also contribute to the observed effects of MG132 in honeybees. However, in the honeybee E64, a specific inhibitor of calpain and the lysosomal proteolysis inhibits midterm memory retention (3-6 h after initial learning) but did not affect long-term memory retention (Grünbaum and Müller 1998). Because we see an enhancement of long-term memory formation after initial learning, we conclude that MG132 in our study targets the proteasome rather than calpain and cathepsin. 
The MGl32-dependent enhancement of memory formation after initial learning depends on the number of trials and the intertrial interval

We demonstrate that the sensitivity of memory formation after initial learning to MG132 depends on the number of trials and the ITI.

In honeybees, the number of trials is critical for protein synthesis-dependent memory formation. After three acquisition trials but not after one trial protein synthesis-dependent memory is formed (Wüstenberg et al. 1998; Friedrich et al. 2004; Stollhoff et al. 2005; Felsenberg et al. 2011). We here reveal an enhancement of memory formation by MG132 after initial learning with three trials but not with one trial. Thus, we observe sensitivity to MG132 after learning protocols that have been demonstrated to induce protein synthesis-dependent consolidation processes. This might indicate that mechanisms underlying the trialdependent activation of protein synthesis simultaneously play a role in protein degradation. This interpretation is in line with the notion that protein synthesis and protein degradation are tightly coupled to establish long-lasting synaptic plasticity (Ashraf et al. 2006; Fonseca et al. 2006; Hou et al. 2006; Karpova et al. 2006; Merlo and Romano 2007; Dong et al. 2008; Banerjee et al. 2009) and the formation of consolidated memory (Ashraf et al. 2006; Merlo and Romano 2007; Kottler et al. 2011).

Furthermore, our study reveals that the ITI during initial learning is critical for the MG132 sensitivity of memory formation after initial learning and extinction learning. Previous studies in honeybees demonstrated that the ITI is critical for long-term memory formation (Gerber et al. 1998; Menzel et al. 2001; Giurfa et al. 2009) and determined the molecular processes underlying memory formation and thus the development of a certain memory phase (Menzel et al. 2001). This holds true also for the fruit fly Drosophila melanogaster, where consecutive conditioning sessions result in long-term memories that differ in terms of stability and protein synthesis dependency depending on the ITI (Tully et al. 1994; Isabel et al. 2004). Interestingly, the highly conserved ubiquitin ligase Neuralized (Neur) enhances long-term memory formation in a dose-dependent and ITI-dependent manner (Pavlopoulos et al. 2008). Hence, the effect of overexpressing Neur on long-term memory formation in D. melanogaster is comparable to an ITI-dependent enhancement of long-term memory formation in the honeybee by the application of the proteasome inhibitor MG132. The amount of Neur is regulated by the proteasome (Deblandre et al. 2001; Pavlopoulos et al. 2008). Thus, it might well be that in the honeybee, Neur is a substrate of an ITI-dependent protease activity.

\section{MG132 sensitivity after initial learning and extinction learning depends in the same way on the learning parameters presented during initial learning}

Our study reveals that the sensitivity of memory formation after extinction learning and initial learning to MG132 depends in the same way on learning parameters like the number of trials and the ITI. This result might indicate that protein degradation after initial learning is a prerequisite for protein degradation after extinction learning, i.e., that both processes are functionally linked. Previously, we demonstrated that protein synthesis-dependent memory consolidation after extinction learning depends on the learning parameter during initial learning, i.e., the reward duration (Stollhoff and Eisenhardt 2009). Our present findings extend this result by showing that the number of trials and the intertrial interval during initial learning are critical for the molecular mechanisms underlying memory consolidation after extinction learning. Accordingly, protein degradation might be one mecha- nism that mediates the dependency of extinction memory consolidation on the learning parameters of initial learning.

\section{Materials and Methods}

The experiments were conducted from May to August 2009, from April to September 2010, and in April 2011.

Animals were treated, conditioned, and systemically injected with $1 \mu \mathrm{L}$ of $0.01 \%$ DMSO/PBS or $1 \mu \mathrm{L}$ of $1 \mu \mathrm{M}$ MG132 (SigmaAldrich) dissolved in $0.01 \%$ DMSO/PBS (PBS: $137 \mathrm{mM} \mathrm{NaCl}$, $2.7 \mathrm{mM} \mathrm{KCl}, 10.1 \mathrm{mM} \mathrm{Na}_{2} \mathrm{HPO}_{4}, 1.8 \mathrm{mM} \mathrm{KH}_{2} \mathrm{PO}_{4}$ at pH 7.2) $1 \mathrm{~h}$ after the onset of acquisition or extinction, as previously described (Stollhoff et al. 2005; Felsenberg et al. 2011). In short, $1 \mathrm{~d}$ before the experiment starts, bees leaving the hive were caught. On the next day, during acquisition, 1, 2, or 3 CS-US trials were presented with an intertrial interval (ITI) of $2 \mathrm{~min}$ or $10 \mathrm{~min}$. One day after acquisition, animals were subjected to extinction. During extinction, two CS-only trials were presented with an ITI of $10 \mathrm{~min}$. During the retention tests, one CS-only trial was presented.

For statistical analysis of multiple comparisons, an analysis of variance for repeated measurement was used (rmANOVA). The critical conditions to use an ANOVA for dichotomous data were met (Lunney 1970). First, the degree of freedom of error was higher than 40, and second, the sample size was equal. To achieve equal sample size despite the loss of animals over the experimental days, 10 random samples were drawn from the bigger group and tested against the smaller group. Only if all 10 comparisons were significantly different were the results treated as significantly different.

For pairwise comparisons of CRs between groups, a G-test for contingency tables (log-likelihood ratio for contingency tables) was used. For the within-group comparison between the CR at the first CS-only trial and the CR at each retention test, we used the McNemar $\chi^{2}$ test.

\section{Acknowledgments}

This study was supported through grant 01GQ0941 to D.E. from the German Federal Ministry of Education and Research (BMBF) within the Bernstein Focus Neuronal Basis of Learning.

\section{References}

Artinian J, McGauran A-MT, De Jaeger X, Mouledous L, Frances B, Roullet P. 2008. Protein degradation, as with protein synthesis, is required during not only long-term spatial memory consolidation but also reconsolidation. Eur J Neurosci 27: 3009-3019.

Ashraf SI, McLoon AL, Sclarsic SM, Kunes S. 2006. Synaptic protein synthesis associated with memory is regulated by the RISC pathway in Drosophila. Cell 124: 191-205.

Banerjee S, Neveu P, Kosik KS. 2009. A coordinated local translational control point at the synapse involving relief from silencing and MOV10 degradation. Neuron 64: 871-884.

Bitterman ME, Menzel R, Fietz A, Schafer S. 1983. Classical conditioning of proboscis extension in honeybees (Apis mellifera). J Comp Psychol 97: $107-119$.

Bouton ME. 2004. Context and behavioral processes in extinction. Learn Mem 11: 485-494.

Chain DG, Casadio A, Schacher S, Hegde AN, Valbrun M, Yamamoto N, Goldberg AL, Bartsch D, Kandel ER, Schwartz JH. 1999. Mechanisms for generating the autonomous cAMP-dependent protein kinase required for long-term facilitation in Aplysia. Neuron 22: 147-156.

Deblandre GA, Lai EC, Kintner C. 2001. Xenopus Neuralized is a ubiquitin ligase that interacts with XDelta1 and regulates Notch signaling. Dev Cell 1: 795-806.

Dong C, Upadhya SC, Ding L, Smith TK, Hegde AN. 2008. Proteasome inhibition enhances the induction and impairs the maintenance of late-phase long-term potentiation. Learn Mem 15: 335-347.

Dudai Y. 2004. The neurobiology of consolidations, or, how stable is the engram? Annu Rev Psychol 55: 51-86.

Felsenberg J, Gehring KB, Antemann V, Eisenhardt D. 2011. Behavioural pharmacology in classical conditioning of the proboscis extension response in honeybees (Apis mellifera). J Vis Exp 2011: 2282. doi: $10.3791 / 2282$.

Fonseca R, Vabulas RM, Hartl FU, Bonhoeffer T, Nagerl UV. 2006. A balance of protein synthesis and proteasome-dependent degradation determines the maintenance of LTP. Neuron 52: 239-245. 
Friedrich A, Thomas U, Muller U. 2004. Learning at different satiation levels reveals parallel functions for the cAMP-protein kinase A cascade in formation of long-term memory. J Neurosci 24: 4460-4468.

Gerber B, Wustenberg D, Schutz A, Menzel R. 1998. Temporal determinants of olfactory long-term retention in honeybee classical conditioning: Nonmonotonous effects of the training trial interval. Neurobiol Learn Mem 69: 71-78.

Giurfa M, Sandoz JC. 2012. Invertebrate learning and memory: Fifty years of olfactory conditioning of the proboscis extension response in honeybees. Learn Mem 19: 54-66.

Giurfa M, Fabre E, Flaven-Pouchon J, Groll H, Oberwallner B, Vergoz V, Roussel E, Sandoz JC. 2009. Olfactory conditioning of the sting extension reflex in honeybees: Memory dependence on trial number, interstimulus interval, intertrial interval, and protein synthesis. Learn Mem 16: 761-765.

Grünbaum L, Müller U. 1998. Induction of a specific olfactory memory leads to a long-lasting activation of protein kinase $\mathrm{C}$ in the antennal lobe of the honeybee. J Neurosci 18: 4384-4392.

Hou L, Antion MD, Hu D, Spencer CM, Paylor R, Klann E. 2006. Dynamic translational and proteasomal regulation of fragile X mental retardation protein controls mGluR-dependent long-term depression. Neuron 51: $441-454$

Imai N, Matsumoto S, Kang W. 2005. Formation of Bombyx mori nucleopolyhedrovirus IE2 nuclear foci is regulated by the functional domains for oligomerization and ubiquitin ligase activity. J Gen Virol 86: $637-644$

Isabel G, Pascual A, Preat T. 2004. Exclusive consolidated memory phases in Drosophila. Science 304: 1024-1027.

Jarome TJ, Werner CT, Kwapis JL, Helmstetter FJ. 2011. Activity dependent protein degradation is critical for the formation and stability of fear memory in the amygdala. PLOS ONE 6: e24349. doi: 10.1371/ journal.pone.0024349.

Karpova A, Mikhaylova M, Thomas U, Knopfel T, Behnisch T. 2006. Involvement of protein synthesis and degradation in long-term potentiation of Schaffer collateral CA1 synapses. J Neurosci 26: 4949-4955.

Katsuma S, Tsuchida A, Matsuda-Imai N, Kang W, Shimada T. 2011. Role of the ubiquitin-proteasome system in Bombyx mori nucleopolyhedrovirus infection. J Gen Virol 92: 699-705.

Kisselev AF, Goldberg AL. 2001. Proteasome inhibitors: From research tools to drug candidates. Chem Biol 8: 739-758.

Kottler B, Lampin-Saint-Amaux A, Comas D, Preat T, Goguel V. 2011. Debra, a protein mediating lysosomal degradation, is required for long-term memory in Drosophila. PLOS ONE 6: e25902. doi: 10.1371/ journal.pone.0025902.

Lattal KM, Radulovic J, Lukowiak K. 2006. Extinction: Does it or doesn't it? The requirement of altered gene activity and new protein synthesis. Biol Psychiatry 60: 344-351.

Lee SH, Choi JH, Lee N, Lee HR, Kim JI, Yu NK, Choi SL, Kim H, Kaang BK. 2008. Synaptic protein degradation underlies destabilization of retrieved fear memory. Science 319: 1253-1256.
Lopez-Salon M, Alonso M, Vianna MR, Viola H, Mello e Souza T, Izquierdo I, Pasquini JM, Medina JH. 2001. The ubiquitin-proteasome cascade is required for mammalian long-term memory formation. Eur J Neurosci 14: 1820-1826.

Lundgren J, Masson P, Mirzaei Z, Young P. 2005. Identification and characterization of a Drosophila proteasome regulatory network. Mol Cell Biol 25: 4662-4675.

Lunney GH. 1970. Using analysis of variance with a dichotomous dependent variable: An empirical study. Int J Educ Meas 7: 263-269.

Menzel R, Manz G, Greggers U. 2001. Massed and spaced learning in honeybees: The role of CS, US, the intertrial interval, and the test interval. Learn Mem 8: 198-208.

Merlo E, Romano A. 2007. Long-term memory consolidation depends on proteasome activity in the crab Chasmagnathus. Neuroscience 147: 46-52.

Myers KM, Davis M. 2002. Behavioral and neural analysis of extinction. Neuron 36: 567-584.

Nader K, Hardt O. 2009. A single standard for memory: The case for reconsolidation. Nat Rev Neurosci 10: 224-234.

Naidoo N, Song W, Hunter-Ensor M, Sehgal A. 1999. A role for the proteasome in the light response of the timeless clock protein. Science 285: $1737-1741$.

Pavlopoulos E, Anezaki M, Skoulakis EM. 2008. Neuralized is expressed in the $\alpha / \beta$ lobes of adult Drosophila mushroom bodies and facilitates olfactory long-term memory formation. Proc Natl Acad Sci 105: $14674-14679$.

Rodriguez-Ortiz CJ, Balderas I, Saucedo-Alquicira F, Cruz-Castaneda P, Bermudez-Rattoni F. 2011. Long-term aversive taste memory requires insular and amygdala protein degradation. Neurobiol Learn Mem 95: $311-315$.

Stollhoff N, Eisenhardt D. 2009. Consolidation of an extinction memory depends on the unconditioned stimulus magnitude previously experienced during training. J Neurosci 29: 9644-9650.

Stollhoff N, Menzel R, Eisenhardt D. 2005. Spontaneous recovery from extinction depends on the reconsolidation of the acquisition memory in an appetitive learning paradigm in the honeybee (Apis mellifera). $J$ Neurosci 25: 4485-4492.

Tully T, Preat T, Boynton SC, Del Vecchio M. 1994. Genetic dissection of consolidated memory in Drosophila. Cell 79: 35-47.

Wüstenberg D, Gerber B, Menzel R. 1998. Long- but not medium-term retention of olfactory memories in honeybees is impaired by actinomycin D and anisomycin. Eur J Neurosci 10: 2742-2745.

Yeh SH, Mao SC, Lin HC, Gean PW. 2006. Synaptic expression of glutamate receptor after encoding of fear memory in the rat amygdala. $\mathrm{Mol}$ Pharmacol 69: 299-308.

Zhao Y, Hegde AN, Martin KC. 2003. The ubiquitin proteasome system functions as an inhibitory constraint on synaptic strengthening. Curr Biol 13: 887-898.

Received March 6, 2012; accepted in revised form June 7, 2012. 


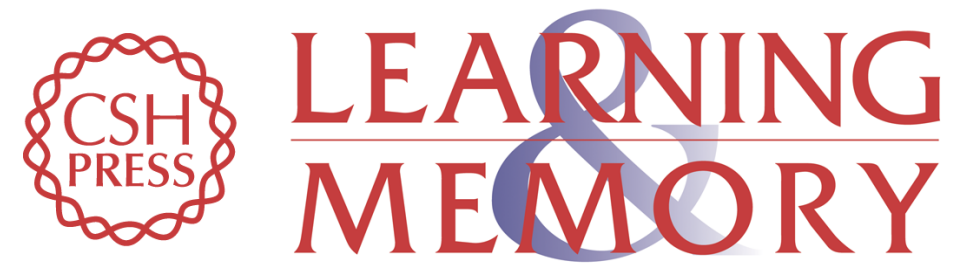

\section{A role of protein degradation in memory consolidation after initial learning and extinction learning in the honeybee (Apis mellifera)}

Johannes Felsenberg, Vincent Dombrowski and Dorothea Eisenhardt

Learn. Mem. 2012, 19:

Access the most recent version at doi:10.1101//m.026245.112

References This article cites 42 articles, 16 of which can be accessed free at:

http://learnmem.cshlp.org/content/19/10/470.full.html\#ref-list-1

License

Email Alerting Receive free email alerts when new articles cite this article - sign up in the box at the Service top right corner of the article or click here. 